\title{
Untangling the Evolutionary History of European Bison (Bison bonasus)
}

Ayla L. van Loenen, Emilia Hofman-Kamińska, Kieren J. Mitchell, Bastien Llamas, Hervé Bocherens, Julien Soubrier, Holly Heiniger, Martina Pacher, Daniel Makowiecki, Giedrè Pilǐciauskienè, Dorothée Drucker, Rafał Kowalczyk, Alan Cooper

This manuscript has been withdrawn by bioRxiv because it was submitted without the consent of all the authors. 